\title{
SPADE: Verification of Multithreaded Dynamic and Recursive Programs ${ }^{\star}$
}

(Tool Paper)

\author{
Gaël Patin ${ }^{1}$, Mihaela Sighireanu ${ }^{2}$, and Tayssir Touili ${ }^{2}$ \\ University of Paris 7, Case 7014, 2 place Jussieu, 75251 Paris 05, France \\ LIAFA, CNRS \& University of Paris 7, Case 7014, 2 place Jussieu, 75251 Paris 05, France \\ \{sighirea, touili\}@liafa.jussieu.fr
}

\section{Introduction}

Recently, there are a lot of tools that have been considered for software verification. We can for example mention BLAST [HJMS02], SLAM [BR01], KISS [QW04QR05], ZING [QRR04], and MAGIC [CCG $\left.{ }^{+} 03 \mathrm{CCG}^{+} 04 \mathrm{CCK}^{+} 06\right]$. However, none of these tools can deal with parallelism, communication between parallel processes, dynamic process creation, and recursion at the same time. The tool we propose, called SPADE, allows to analyse automatically boolean programs presenting all these features. As far as we know, this is the first software model checking tool based on an expressive model that accurately models all these aspects in programs.

SPADE checks safety properties of programs by iteratively refining abstractions of the sets of the program execution paths that violate the property. Since property checking is undecidable for programs presenting all the features mentioned above, the SPADE refinement algorithm may not converge. In case of convergence, it can either find a bug in the program and returns a counterexample to the user, or certify that the program is correct.

We have applied SPADE to different case studies. Our results are encouraging and are reported in Section 4 In particular, we were able to automatically find two bugs in two versions of a Windows NT Bluetooth driver. The bugs were already found in $\left[\mathrm{CCK}^{+} 06\right]$. But there, the verification was not completely automatic since the authors needed to guess the number of processes for which the bugs occur. Whereas with SPADE, the verification process was done in a completely automatic manner. Indeed, we don't need to make any guess since our tool handles dynamic creation of processes.

The current version of SPADE is available at http://www.liafa.jussieu.fr/ sighirea/spade.

\section{The Underlying Techniques}

SPADE is based on the SPAD model [Tou05]. A SPAD is a finite set of rules of the form $t \stackrel{a}{\longrightarrow} t^{\prime}$, where $a$ is a synchronisation action, $t$ and $t^{\prime}$ are terms built up from the

\footnotetext{
* This work has been supported by the French Governement program ACI Jeunes Chercheurs, Contract No.02 20205.
} 
null process "0", a finite number of variables $(X)$, the sequential composition ".", and the asynchroneous parallel composition "||", where the operators "." and "||" are respectively associative and associative/commutative, and where each action $a$ has its corresponding co-action $\bar{a}$. Intuitively, the process "0" represents termination, a process variable $X$ corresponds to a control point of the program, and a process term $t$ describes the control structure of the program. A procedure call is represented by a rule of the form $X \rightarrow Y \cdot Z$, where the program at control point $X$ calls the procedure $Y$ and goes to control point $Z$. This control point $Z$ becomes active when $Y$ terminates. Dynamic creation of parallel processes is modeled by rules of the form $X \rightarrow Y|| Z$, expressing that a process in control point $X$ can create two parallel processes in control points $Y$ and $Z$, respectively. Finally, handshakes between parallel processes are represented according to the CCS style by rules of the form $t_{1} \stackrel{a}{\longrightarrow} t_{1}^{\prime}$ and $t_{2} \stackrel{\bar{a}}{\longrightarrow} t_{2}^{\prime}$, meaning that two parallel processes $t_{1}$ and $t_{2}$ can synchronize and move simultaneously to $t_{1}^{\prime}$ and $t_{2}^{\prime}$, respectively.

SPADE deals with rechability queries for SPAD models. More precisely, given two (possibly infinite) sets of configurations Init and Bad, the problem is to know whether the set of bad configurations Bad can be reached from the initial configurations Init. The approach implemented in SPADE consists in computing abstractions of the execution path language that leads form Init to Bad and iteratively refining these abstractions [Tou05]. Our techniques are based on (1) the representation of the sets of configurations with binary tree automata, (2) the use of these automata to compute a set of constraints whose least fixpoint characterize the set of execution paths of the program, and (3) the resolution of this set of constraints in an abstract domain. Our algorithm is generic and can deal with different abstract domains. In particular, we considered the domains $D_{n}$ of finite action words of length less or equal to $n$. These domains allow to compute abstractions of the execution paths that are exact up to the depth $n$. These abstractions are called $n$-prefix abstractions. The refinement step consists in considering a "more precise" abstract domain by incrementing the depth $n$.

\section{The SPADE Tool}

SPADE has two inputs. The first input is an ASCII file describing (1) the SPAD model of the program (names of processes, names of actions, rewriting rules), (2) the (possibly infinite) set of initial configurations Init (given by a tree automaton), and (3) the bad configuration Bad (a tree automaton). The second input is optional and consists of an integer that represents the depth $n$ of the prefix abstraction. If this parameter is not given by the user, the tool starts with a prefix abstration of depth one, and automatically increases the abstraction depth until either an error is found or the program is proven to be correct.

SPADE outputs (a) the language reach $_{n}$ representing the $n$-prefix abstraction of the paths between Init and $\mathrm{Bad}$, and (b) the result of the intersection of reach ${ }_{n}$ with the set of good execution paths. This result may be either (CANNOT) if the intersection is empty (i.e., the $n$-prefix abstraction does not allow to find an execution leading from Init to Bad), (MAYBE) if the intersection is not empty but the path found has been cut by the abstraction, (CAN) if a real path (i.e., not cut by abstraction) has been found between Init and Bad. 
SPADE implements in OCAML the algorithm described in [Tou05]. OCAML provides a rich and efficient built-in library of data structures (e.g., hash tables, maps, sets), a powerful system of modules, and garbage collection facilities. Due to these features, the algorithm is implemented as a generic module parameterized by two signatures (interfaces): the first signature collects types and operations dealing with tree automata, and the second signature collects types and operations of the abstract domain of execution paths. The current version of SPADE instantiates the first parameter of the algorithm with the OCAML implementation of tree automata provided by the TIMBUK tool [GT01]. This implementation provides a large list of operations on tree automata (union, intersection, emptiness test, minimization, etc) and an easy access to the states and the transitions of automata. For the second parameter, we implemented in OCAML a library for the abstract domain $D_{n}$ (i.e., finite sets of finite words of length less or equal to $n$ ). The library provides efficient implementation of operations intensively used by the algorithm: union, concatenation, shuffle, prefix, and inclusion.

\section{Summary of the Results}

SPADE has been applied to several examples. The performances are given in Table 1 The experiments were obtained on a $4 \mathrm{GHz}$ Pentium IV with 4GB of memory.

Table 1. Performances of SPADE

\begin{tabular}{||c|c|c||}
\hline \hline Example & Time & Space \\
\hline \hline BlueTooth v1 & $1623 \mathrm{mn} 28 \mathrm{~s}$ & $50 \mathrm{MB}$ \\
\hline BlueTooth v2 & $1216 \mathrm{mn} 28 \mathrm{~s}$ & $46 \mathrm{MB}$ \\
\hline \hline ConcVector v1 & $7 \mathrm{~s}$ & $3.4 \mathrm{MB}$ \\
\hline ConcVector v2 & $14 \mathrm{~s}$ & $14.8 \mathrm{MB}$ \\
\hline \hline Lock/unlock & $8 \mathrm{~s}$ & $3.6 \mathrm{MB}$ \\
\hline \hline
\end{tabular}

The BlueTooth $\mathrm{v} 1$ is the SPAD model of the BlueTooth driver program used by Windows NT and given in [QW04]. We were able to find a bug in this program. To find this error, the [QW04] authors needed to guess the number of driver's requests for which the error occurs, and then run their tool; whereas with SPADE, the verification was done in a completely automatic manner, since we did not have to guess the number of requests for which the error occurs because our tool can deal with dynamic creation of processes.

The BlueTooth v2 is a corrected version of BlueTooth v1 proposed by the authors of [QW04]. SPADE finds an error in this version as well. This bug was already found in $\left[\mathrm{CCK}^{+} 06\right]$. Again, to be able to find the bug, the authors of $\left[\mathrm{CCK}^{+} 06\right]$ needed to guess the number of requests that causes the bug before running their tool, whereas SPADE did not need to perform this guess.

ConcVector is a SPAD model of a multithreaded program using concurrently methods of the class java.util.Vector from the Java Standard Collection Framework. The program's threads create and remove the elements of a Vector object. Wand and Stoller [WS03] reported a high-level data race that occurs on such programs because 
the constructor of the Vector class is not atomic. SPADE found this bug for a program with an unbounded number of threads (ConcVector v1). Version v2 fixes the bug by taking an atomic implementation of the constructor. SPADE was able to prove that this version is correct.

The Lock/unlock example is a system that handles an arbitrary number of concurrent insertions on a binary search tree. The algorithm was proposed in [KL80], and can be applied to handle simultaneous insertions (done by several users) into a database, or to reduce the time necessary for a single insertion. We considered a buggy version of the algorithm where one or several processes do not adhere to the required lock and unlock policy. This version was considered in $\left[\mathrm{CCK}^{+} 06\right]$, where the bug was found only for systems where the number of concurrent processes is less or equal to 7. With SPADE, we were able to check this buggy program for arbitrary number of concurrent insertion processes.

\section{References}

BR01. Ball, T., Rajamani, S.K.: Automatically validating temporal safety properties of interfaces. In: Dwyer, M.B. (ed.) Model Checking Software. LNCS, vol. 2057, Springer, Heidelberg (2001)

$\mathrm{CCG}^{+}$03. Chaki, S., Clarke, E., Groce, A., Jha, S., Veith, H.: Modular verification of software components in C. In: International Conference on Software Engineering (ICSE), pp. 385-395 (2003)

$\mathrm{CCG}^{+}$04. Chaki, S., Clarke, E., Grumberg, O., Ouaknine, J., Sharygina, N., Touili, T., Veith, H.: An expressive framework for state/event systems. Technical report, Carnegie Mellon University (2004)

$\mathrm{CCK}^{+}$06. Chaki, S., Clarke, E., Kidd, N., Reps, T., Touili, T.: Verifying concurrent messagepassing C programs with recursive calls. In: TACAS (2006)

GT01. Genet, T., Viet Triem Tong, V.: Reachability analysis of term rewriting systems with timbuk. In: Nieuwenhuis, R., Voronkov, A. (eds.) LPAR 2001. LNCS (LNAI), vol. 2250, pp. 695-706. Springer, Heidelberg (2001)

HJMS02. Henzinger, T.A., Jhala, R., Majumdar, R., Sutre, G.: Lazy abstraction. In: Symposium on Principles of Programming Languages, pp. 58-70 (2002)

KL80. Kung, H.T., Lehman, P.L.: Concurrent manipulation of binary search trees. ACM Trans. Database Syst. 5(3), 354-382 (1980)

QR05. Qadeer, S., Rehof, J.: Context-bounded model checking of concurrent software. In: Halbwachs, N., Zuck, L.D. (eds.) TACAS 2005. LNCS, vol. 3440, Springer, Heidelberg (2005)

QRR04. Qadeer, S., Rajamani, S.K., Rehof, J.: Summarizing procedures in concurrent programs. In: POPL 04: ACM Principles of Programming Languages, pp. 245-255 (2004)

QW04. Qadeer, S., Wu, D.: Kiss: Keep it simple and sequential. In: PLDI 04: Programming Language Design and Implementation, pp. 14-24 (2004)

Tou05. Touili, T.: Dealing with communication for dynamic multithreaded recursive programs. In: 1st VISSAS workshop, Invited Paper (2005)

WS03. Wang, L., Stoller, S.D.: Run-time analysis for atomicity. In: Proceedings of the Third Workshop on Runtime Verification (RV). Electronic Notes in Theoretical Computer Science, vol. 89(2), Elsevier, Amsterdam, Netherlands (2003) 